\title{
Human HemK2/KMT9/N6AMT1 is an active protein methyltransferase, but does not act on DNA in vitro, in the presence of Trm112
}

\author{
Clayton B. Woodcock', Dan Yu' ${ }^{1}$, Xing Zhang ${ }^{1}$ and Xiaodong Cheng ${ }^{1}$
}

Dear Editor,

Ever since the initial discovery and characterization of $S$-adenosyl-L-methionine (SAM $)^{1}$, the ATP-activated form of methionine, SAM has become the second most commonly used enzyme cofactor after ATP. A wide variety of macromolecules, including DNA, RNA, proteins, polysaccharides, lipids, and a range of small molecules are subject to methylation by highly specific SAM-dependent methyltransferases (MTases) acting on a particular target atom. Examples of methylation targets include nucleic acids (cytosine-C5, cytosine-N4 and adenine-N6), protein residues (arginine- $\mathrm{N}$, lysine- $\mathrm{N}$, glutamine- $\mathrm{N}$ and histidine- $\mathrm{N}$ ), and small molecules (catechol-O, histamine- $\mathrm{N}$, glycine- $\mathrm{N}$, and thiopurine-S).

Early sequence alignments of known SAM-dependent MTases identified a set of conserved sequence motifs among DNA cytosine-C5 MTases, DNA adenine-N6 and cytosine-N4 MTases, and protein or small molecule MTases $^{2}$. In essence, the two most conserved sequence motifs reflect the common functions of the MTases: motif I (FxGxG) for binding of the methyl donor SAM and motif IV for catalysis. The residues of motif IV conform in accordance with the target atom, for instance, the PC motif (proline-cysteine) is responsible for ring carbon-C5 methylation of cytosine, whereas the $(\mathrm{D} / \mathrm{N}) \mathrm{PP}(\mathrm{Y} / \mathrm{F} / \mathrm{W})$ motif for exocyclic amino methylation of adenine and cytosine. However, the apparent sequence and structural similarity do not reveal with certainty whether an enzyme acts on a particular substrate (DNA, RNA or protein). Examples include mammalian DNMT2, which shares sequence and structural similarity with DNA cytosine-C5

\footnotetext{
Correspondence: Xing Zhang (xzhang21@mdanderson.org) or

Xiaodong Cheng (xcheng5@mdanderson.org)

${ }^{1}$ Department of Epigenetics and Molecular Carcinogenesis, University of Texas MD Anderson Cancer Center, Houston, TX 77030, USA
}

MTases $^{3}$, but is a tRNA cytosine-C5 MTase; ${ }^{4}$ and Escherichia coli (E. coli) HemK, which was thought to be a DNA amino MTase ${ }^{5}$, yet is a protein glutamine MTase ${ }^{6,7}$. The conservation in an enzyme's sequence and structure merely reflects the conserved nature of catalyzing the methyl transfer onto cytosine-C5 (of DNA and RNA) or amino-group (of adenine and glutamine).

Recently, mammalian HemK2 (NCBI Reference Sequence NP_037372.4) has been documented to be a DNA adenine-N6 MTase ${ }^{8}$ (renamed as N6AMT1) and a histone H4 lysine-12 MTase $^{9}$ (renamed as KMT9), in addition to its known activity of glutamine methylation of eukaryotic release factor $\mathrm{eRF} 1^{10}$. The common feature of the three potential substrates is the amino group $\left(\mathrm{NH}_{2}\right)$ of glutamine, adenine, and lysine (Fig. 1a). It is rare to have a MTase (or any enzyme per se) capable of acting on both oligonucleotides and protein (and on two different amino acids). Because previous studies ${ }^{8,9}$ did not compare directly the three potential substrates, here we analyze the in vitro activities of HemK2 on the three substrates sideby-side. HemK2 forms a heterodimeric complex with $\operatorname{Trm} 112^{10}$ (named after tRNA methylation protein), and we purified recombinant human HemK2-Trm112 heterodimer expressed in E. coli (Fig. 1b). Sequence of one of the characterized DNA substrates (GAGTC) of HemK2 ${ }^{8}$ overlaps with the recognition sequences (GANTC) of a known bacterial DNA adenine MTase, Caulobacter crescentus cell cycle-regulated MTase ${ }^{11}$, we thus included CcrM as a positive control. In addition, we included E. coli Dam (DNA adenine MTase) on GATC for comparison ${ }^{12}$. Both bacterial enzymes were purified in house (Fig. 1b).

We designed five short DNA double-stranded oligos (\#1 to \#5): \#1 contains no-adenine and was used as a negative control, \#2, \#3 and \#4 oligos are thought to be substrates of HemK2 ${ }^{8}, \# 4$ contains the CcrM recognition sequence 


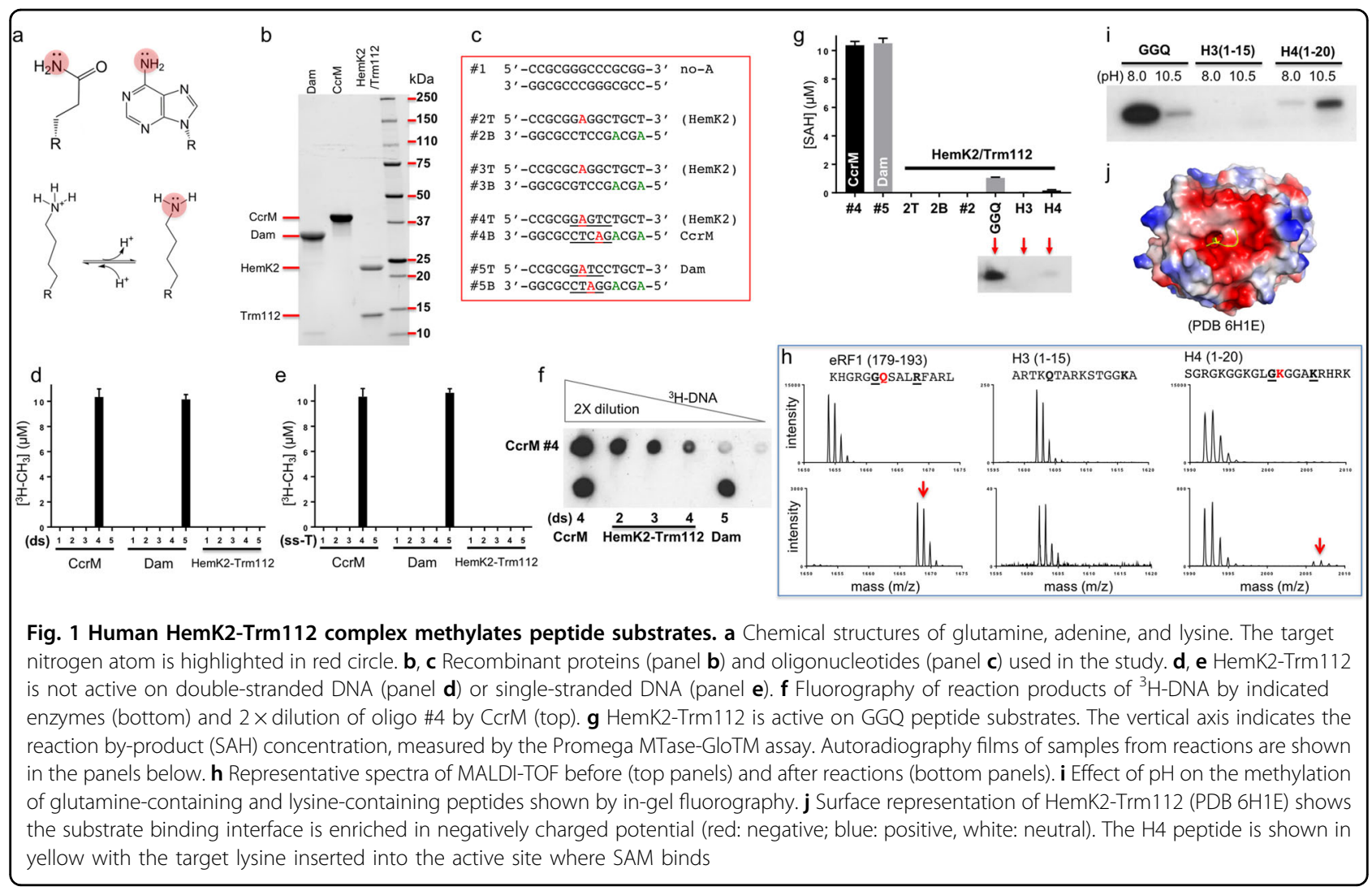

GANTC and \#5 contains the E. coli Dam recognition sequence GATC (Fig. 1c-the letters T and B designate the top and the bottom strand respectively). Under the conditions where both CcrM and Dam completed reactions on their respective substrates (CcrM on oligo \#4 and Dam on oligo \#5), we observed no activities of HemK2Trm112 on the five oligos examined (either double or single stranded) (Fig. 1d-f). However, we did observe activity of HemK2-Trm112 on peptide derived from eRF1 (residues 179-193), much-reduced activity on histone H4 (residues 1-20), and no activity on histone $\mathrm{H} 3$ peptide (residues 1-15) at the condition of $\mathrm{pH} 8$ (Fig. 1g). Under the same condition of $\mathrm{pH} 8$, mass spectrometry analysis of peptide substrates confirmed that glutamine-containing eRF1 peptides were fully methylated, whereas $\mathrm{H} 4$ peptides had residual methylated species and no methylation of $\mathrm{H} 3$ peptides (Fig. 1h).

Most (if not all) SAM-dependent MTases use the classic $\mathrm{S}_{\mathrm{N}} 2$ reaction mechanism, which requires the target nitrogen be in a deprotonated state. Under the condition $(\mathrm{pH}$ 8.0) that HemK2-Trm112 demonstrated strong activity for the glutamine-containing peptide, we observed minor activity on the lysine-containing $\mathrm{H} 4$ peptide (Fig. 1i). We reasoned that higher $\mathrm{pH}$ conditions might enhance methylation of lysine substrate (with typical pKa value of $\sim 10$ ), as was shown for other biochemically characterized protein (histone) lysine MTases to have optimal in vitro activity at approximately $\mathrm{pH} 10^{13}$. After adjusting the $\mathrm{pH}$ to 10.5 , the activity of HemK2-Trm112 is significantly increased on $\mathrm{H} 4$ peptide (Fig. 1i). An investigation employing peptide array libraries showed that HemK2 methylation activity on eRF1 peptide requires a glycine immediately before and an arginine positioned four-residues after the target glutamine ${ }^{14}$. H4 K12 meets this requirement with glycine (G11) and a positively charged lysine (K16) in the place of the corresponding arginine of eRF1 (see sequences shown in Fig. 1h). The short H3 peptide (residues 1-15) used in the assay does contain one glutamine and three lysine residues but is not a methylation substrate of HemK2. Finally, HemK2-Trm112 complex structure (PDB 6H1E ${ }^{9}$ ) illustrates a negatively charged substrate interface (Fig. 1j), ideal for positively charged peptides such as eRF1 and histone $\mathrm{H} 4$, but unsuitable for a negatively charged DNA substrate. We note that the activity on glutaminecontaining eRF1 peptide is significantly reduced at the high $\mathrm{pH}$ (Fig. 1i), suggesting that high $\mathrm{pH}$ is not optimal for catalysis and/or stability of HemK2-Trm112 complex. For the lysine-containing H4 peptide, however, deprotonation of lysine by high $\mathrm{pH}$ is preferred for methylation to occur, but only to a much-reduced level as compared with that of glutamine methylation at $\mathrm{pH} 8$. 
In sum, using two established MTase assays in vitro, following either incorporation of tritium from ${ }^{3} \mathrm{H}-\mathrm{SAM}$ into a substrate or formation of byproduct $\mathrm{SAH}$ in a bioluminescence assay, we show that human HemK2Trm112 heterodimer is only active on protein glutamine and lysine, but not active on DNA. In light of current interests on N6-methyladenine in eukaryotic genomic DNA, our study resolves one controversy in the field. Additional data will be required to address the dual specificity of HemK2 on protein glutamine and lysine-a unique property for a MTase working on two different substrates with different target residues, one is located in the cytoplasm (eRF1, glutamine) and the other in nucleus (histone $\mathrm{H} 4$, lysine).

Finally, we want to clearly highlight the differences between our studies presented here and that of Xiao et al. ${ }^{8}$. (1) We used recombinant HemK2-Trm112 complex expressed in E. coli and purified using a four-column chromatography, whereas Xiao et al. used N6AMT1-Flag plasmid transfected into HEK293T cells and protein purified according to the kit instructions, presumably one-step affinity purification. (2) We used purified HemK2-Trm112 complex (Fig. 1b), whereas there was no mention whether the protein preparation used by Xiao et al. contains Trm112. In a separate study, partially purified Flag-N6AMT1 from overexpressing HEK293T cells appeared to contain a $\sim 15-\mathrm{kDa}$ protein that might be co-purified endogenous Trm112, and this protein preparation did not methylate DNA oligonucleotides in vitro as shown by N6mA specific antibody (see Fig. S4 of Xie et al. ${ }^{15}$ ). (3) Our fluorography assays on DNA (Fig. 1f) were conducted with $[\mathrm{E}]=2 \mu \mathrm{M},[\mathrm{S}]=$ $10 \mu \mathrm{M}$, and $\left[{ }^{3} \mathrm{H}-\mathrm{SAM}\right]=10 \mu \mathrm{M}$, whereas Xiao et al. used $25 \mu \mathrm{l}$ reaction system containing $[\mathrm{E}]=0.8 \mu \mathrm{g}(\sim 0.8 \mu \mathrm{M})$, $\left[\mathrm{S}_{\mathrm{DNA}}\right]=250 \mathrm{pmol}(=10 \mu \mathrm{M}),\left[{ }^{3} \mathrm{H}-\mathrm{SAM}\right]=55 \mu \mathrm{M}$ (which is puzzling as that is the concentration of the PerkinElmer NET155V [ $\left.{ }^{3} \mathrm{H}-\mathrm{SAM}\right]$ stock). Both reactions were carried out at $25^{\circ} \mathrm{C}$ overnight under similar buffer and salt conditions.

\section{Acknowledgements}

This work was supported by grants from NIH (GM049245) and CPRIT (RR160029). The Proteomics and Metabolomics Facilities were supported in part by CPRIT (RP130397) and NIH (1S10OD012304 and P30CA016672). We thank Dr. John R. Horton for providing purified E. coli Dam.

\section{Author contributions}

C.B.W. performed protein purification and enzymatic assays. D.Y. made Trm112 construct. X.Z. and X.C. organized and designed the scope of the study. All were involved in analyzing data and preparing the manuscript.
Conflict of interest

The authors declare that they have no conflict of interest.

\section{Publisher's note}

Springer Nature remains neutral with regard to jurisdictional claims in published maps and institutional affiliations.

Supplementary Information accompanies the paper at (https://doi.org/ 10.1038/s41421-019-0119-5).

Received: 27 June 2019 Accepted: 6 August 2019

Published online: 10 September 2019

\section{References}

1. Cantoni, G. L. The nature of the active methyl donor formed enzymatically from I-methionine and adenosinetriphosphate. J. Am. Chem. Soc. 74, 2942-2943 (1952).

2. Malone, T., Blumenthal, R. M. \& Cheng, X. Structure-guided analysis reveals nine sequence motifs conserved among DNA amino-methyltransferases, and suggests a catalytic mechanism for these enzymes. J. Mol. Biol. 253, 618-632 (1995).

3. Dong, A. et al. Structure of human DNMT2, an enigmatic DNA methyltransferase homolog that displays denaturant-resistant binding to DNA. Nucleic Acids Res. 29, 439-448 (2001).

4. Goll, M. G. et al. Methylation of tRNAAsp by the DNA methyltransferase homolog Dnmt2. Science 311, 395-398 (2006).

5. Bujnicki, J. M. \& Radlinska, M. Is the Hemk family of putative Sadenosylmethionine-dependent methyltransferases a "missing" zeta subfamily of adenine methyltransferases? A hypothesis. IUBMB Life 48, 247-249 (1999).

6. Nakahigashi, K. et al. HemK, a class of protein methyl transferase with similarity to DNA methyl transferases, methylates polypeptide chain release factors, and hemK knockout induces defects in translational termination. Proc. Natl Acad. Sci. USA 99, 1473-1478 (2002).

7. Heurgue-Hamard, V., Champ, S., Engstrom, A., Ehrenberg, M. \& Buckingham, R. H. The hemK gene in Escherichia coli encodes the N(5)-glutamine methyltransferase that modifies peptide release factors. EMBO J. 21, 769-778 (2002).

8. Xiao, C. L. et al. N(6)-methyladenine DNA modification in the human genome. Mol. Cell 71, 306-318 (2018).

9. Metzger, E. et al. KMT9 monomethylates histone H4 lysine 12 and controls proliferation of prostate cancer cells. Nat. Struct. Mol. Biol. 26, 361-371 (2019).

10. Figaro, S., Scrima, N., Buckingham, R. H. \& Heurgue-Hamard, V. HemK2 protein, encoded on human chromosome 21, methylates translation termination factor eRF1. FEBS Lett. 582, 2352-2356 (2008).

11. Zweiger, G., Marczynski, G. \& Shapiro, L. A Caulobacter DNA methyltransferase that functions only in the predivisional cell. J. Mol. Biol. 235, 472-485 (1994).

12. Horton, J. R., Liebert, K., Hattman, S., Jeltsch, A. \& Cheng, X. Transition from nonspecific to specific DNA interactions along the substrate-recognition pathway of dam methyltransferase. Cell 121, 349-361 (2005).

13. Zhang, X. et al. Structure of the Neurospora SET domain protein DIM-5, a histone H3 lysine methyltransferase. Cell 111, 117-127 (2002).

14. Kusevic, D., Kudithipudi, S. \& Jeltsch, A. Substrate specificity of the HEMK2 protein glutamine methyltransferase and identification of novel substrates. J. Biol. Chem. 291, 6124-6133 (2016).

15. Xie, Q. et al. N(6)-methyladenine DNA Modification in Glioblastoma. Cell 175, 1228-1243 (2018) 